\title{
Long-Term and Short-Term Prognostic Value of Circulating Soluble Suppression of Tumorigenicity-2 Concentration in Chronic Heart Failure: A Systematic Review and Meta-Analysis
}

\author{
Guoqi Dong Hao Chen Hongru Zhang Yihuang Gu
}

School of Acupuncture and Tuina, Nanjing University of CM, Nanjing, China

\section{Keywords}

Chronic heart failure . Soluble suppression of tumorigenicity-2 Prognosis

\begin{abstract}
Introduction: Soluble suppression of tumorigenicity-2 (sST2) has been considered as a prognostic factor of cardiovascular disease. However, the prognostic value of SST2 concentration in chronic heart failure remains to be summarized. Methods: We searched PubMed, Embase, and Web of Science for eligible studies up to January 1, 2020. Data extracted from articles and provided by authors were used in agreement with the PRISMA statement. The endpoints were all-cause mortality (ACM), cardiovascular mortality (CVM)/ heart failure-related hospitalization (HFH), and all-cause mortality (ACM)/heart failure-related readmission (HFR). $\boldsymbol{R e}$ sults: A total of 11 studies with 5,121 participants were included in this analysis. Higher concentration of SST2 predicted the incidence of long-term ACM (hazard ratio [HR]: 1.03, 95\% confidence interval [Cl]: 1.02-1.04), long-term ACM/ HFR (HR: 1.42, Cl: 1.27-1.59), and long-term CVM/HFH (HR: 2.25, Cl: 1.82-2.79), regardless of short-term ACM/HFR (HR:
\end{abstract}

karger@karger.com

(c) 2021 S. Karger AG, Basel

www.karger.com/crd

Karger'
2.31, Cl: 0.71-7.49). Conclusion: Higher SST2 concentration at baseline is associated with increasing risk of long-term ACM, ACM/HFR, and CVM/HFH and can be a tool for the prognosis of chronic heart failure.

(c) 2021 S. Karger AG, Basel

\section{Introduction}

Heart failure is the terminal stage of various heart diseases, with high all-cause mortality and heart failure-related readmission [1], and increasing number of patients aggravate the global economic burden [2]. In the stage of chronic heart failure (CHF), the heart of a human has a remarkable degree of plasticity [3], and ventricular remodeling is an important sign of heart failure. Cardiac fibroblasts, upon injury, will transform to a myofibroblast phenotype, which contributes to cardiac fibrosis [4]. Soluble suppression of tumorigenicity-2 (sST2) blocks the cardioprotective effects of interleukin-33 (IL33) in the way of sequestering its availability to bind with the ST2L receptor. In this way, sST2 has been proved to be the marker of myocardial fibrosis and heart failure 
Fig. 1. Flowchart of the selection procedure. CHF, chronic heart failure.

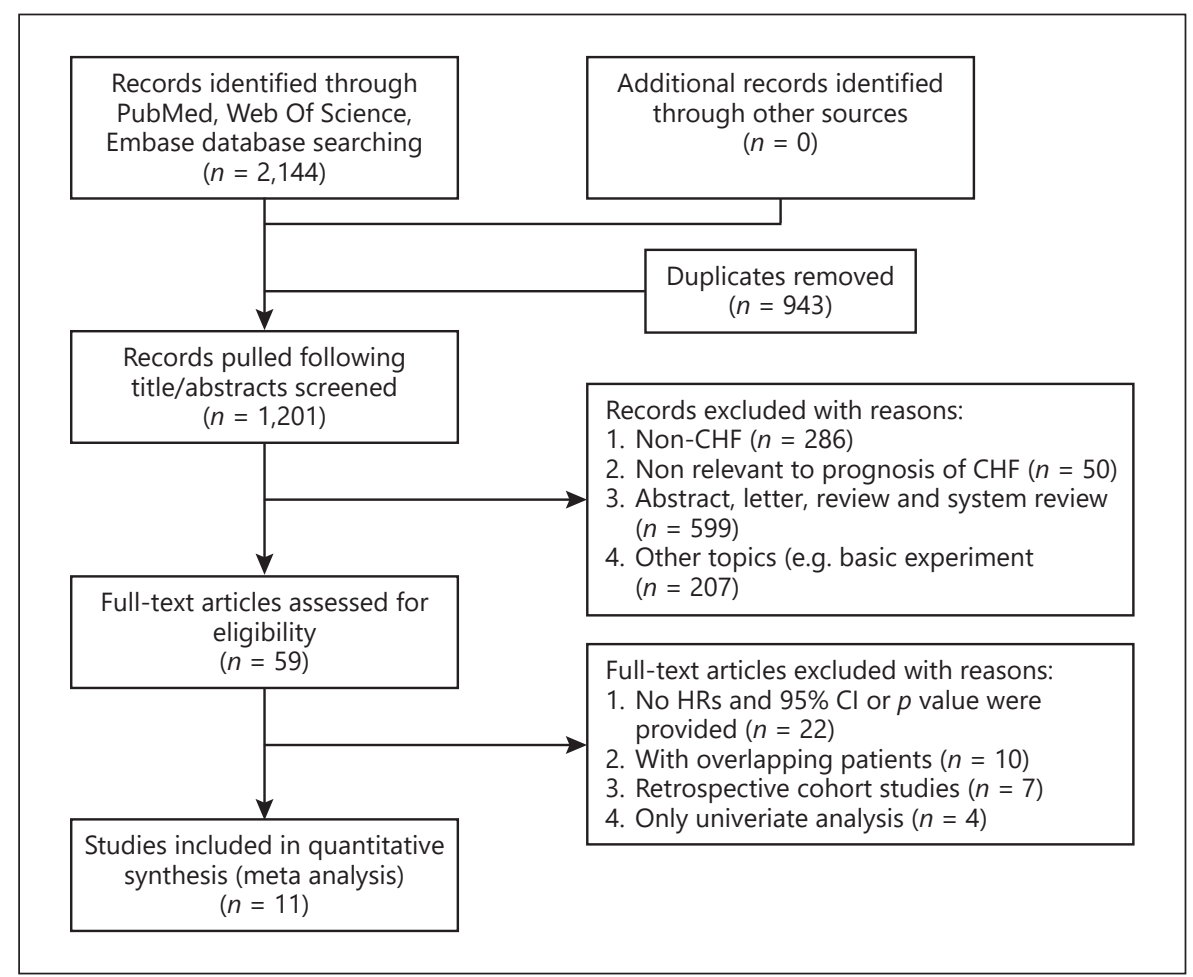

progression [5] and has potential to be the prognosis index of heart failure, which is valuable for further study [6]. Although many studies have suggested that higher concentration of sST2 in baseline is related to a higher rate of HF events, most of them [7-17] vary in follow-up duration. In this paper, meta-analysis is used to provide evidence-based basis for the clinical prognosis evaluation of sST2 for long-term and short-term outcomes in CHF patients.

\section{Methods}

This meta-analysis was performed in accordance with MOOSE (Meta-analysis of Observational Studies in Epidemiology) [18].

\section{Database Search}

Two investigators (G.D. and H.C.) conducted a systematic search of PubMed, Embase, and Web of Science for eligible studies up to January 1, 2020, using the following keywords: ("heart failure" OR "Cardiac Failure" OR "Myocardial Failure" OR "Congestive Heart Failure" OR "Heart Decompensation" OR "Left-Sided Heart Failure" OR "Left Sided Heart Failure" OR "Right-Sided Heart Failure" OR "Right Sided Heart Failure" OR "cardiac dysfunction" OR "cardiac insufficiency" OR "left ventricular dysfunction") AND ("ST2" OR "suppression of tumorigenicity 2").

\section{Inclusion and Exclusion Criteria}

Studies fulfilling the following criteria were included: (1) English language; (2) cohort studies, including prospective studies or post hoc analysis of randomized controlled trials; (3) the concentration of sST2 measured at baseline; (4) patients diagnosed with $\mathrm{HF}$ and aged over 18 years; and (5) reporting the adjusted hazard ratios (HRs) and corresponding 95\% confidence intervals (CIs) for the risk of all-cause mortality (ACM), all-cause mortality/heart failure-related readmission (ACM/HFR), and cardiovascular mortality/heart failure-related hospitalization (CVM/HFH). Retrospective studies, overlapping studies, and studies with incomplete basic information of patients were excluded. Two authors independently included studies, and the disagreement was resolved by discussion.

\section{Data Extraction and Quality Evaluation}

Data extraction was performed by 2 authors (G.D. and H.C.), and disagreement about data was resolved by discussion. The following data of the included studies were extracted: author, publication year, study location, study design, sample size, basic clinical information (mean ages and proportions of males), follow-up durations, variables which were adjusted, $\mathrm{HR}, 95 \% \mathrm{CI}$, and the cutoff value of sST2 concentration. For studies not providing HRs or $95 \%$ CI, useful data would be extracted from survival distributions [19]. Two authors evaluated the quality of studies independently with the Newcastle-Ottawa Scale [20].

Statistical Analyses

All the references of studies were imported into the EndNote software (version X9). Our study used HR to measure the prognos- 


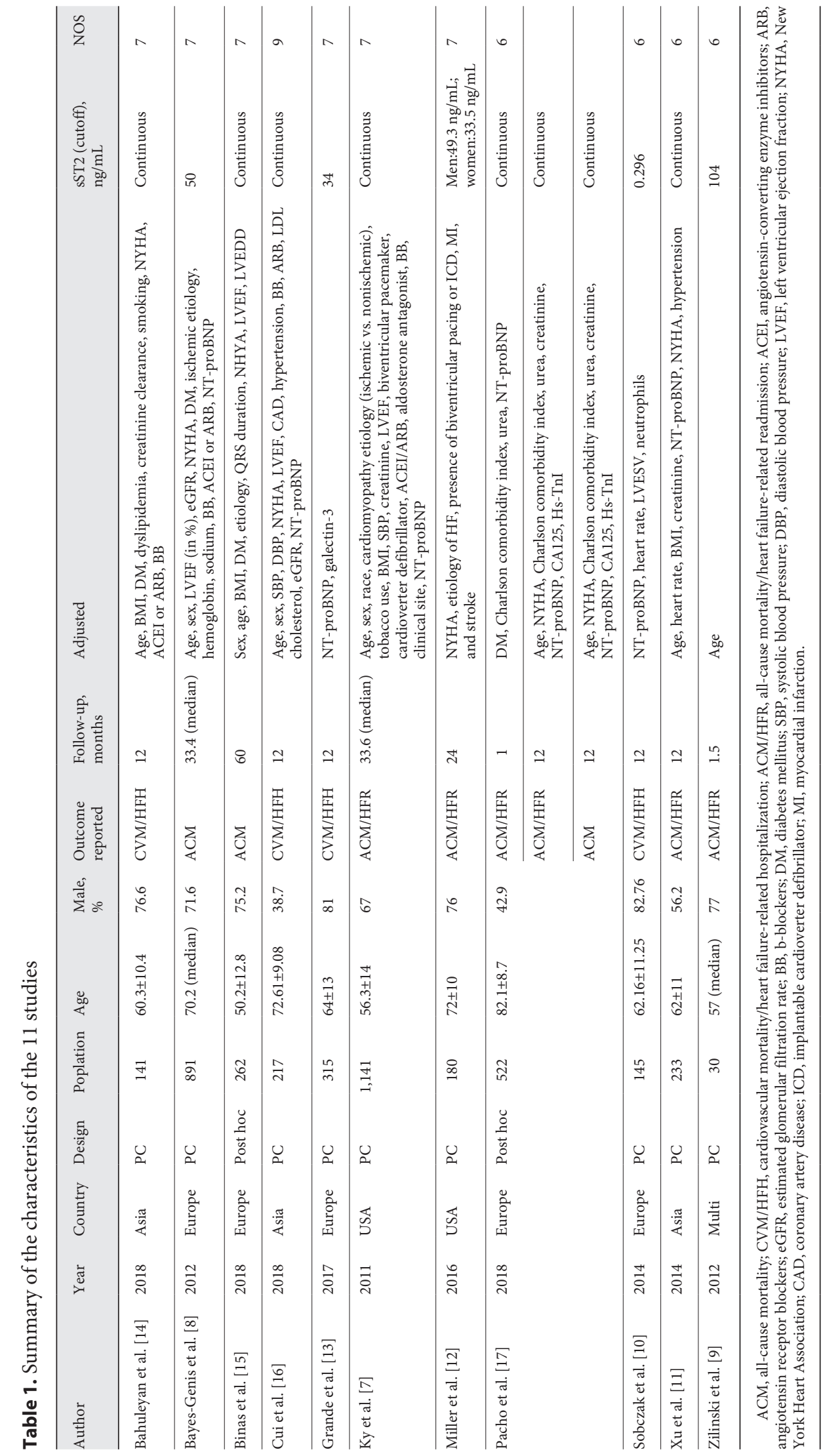




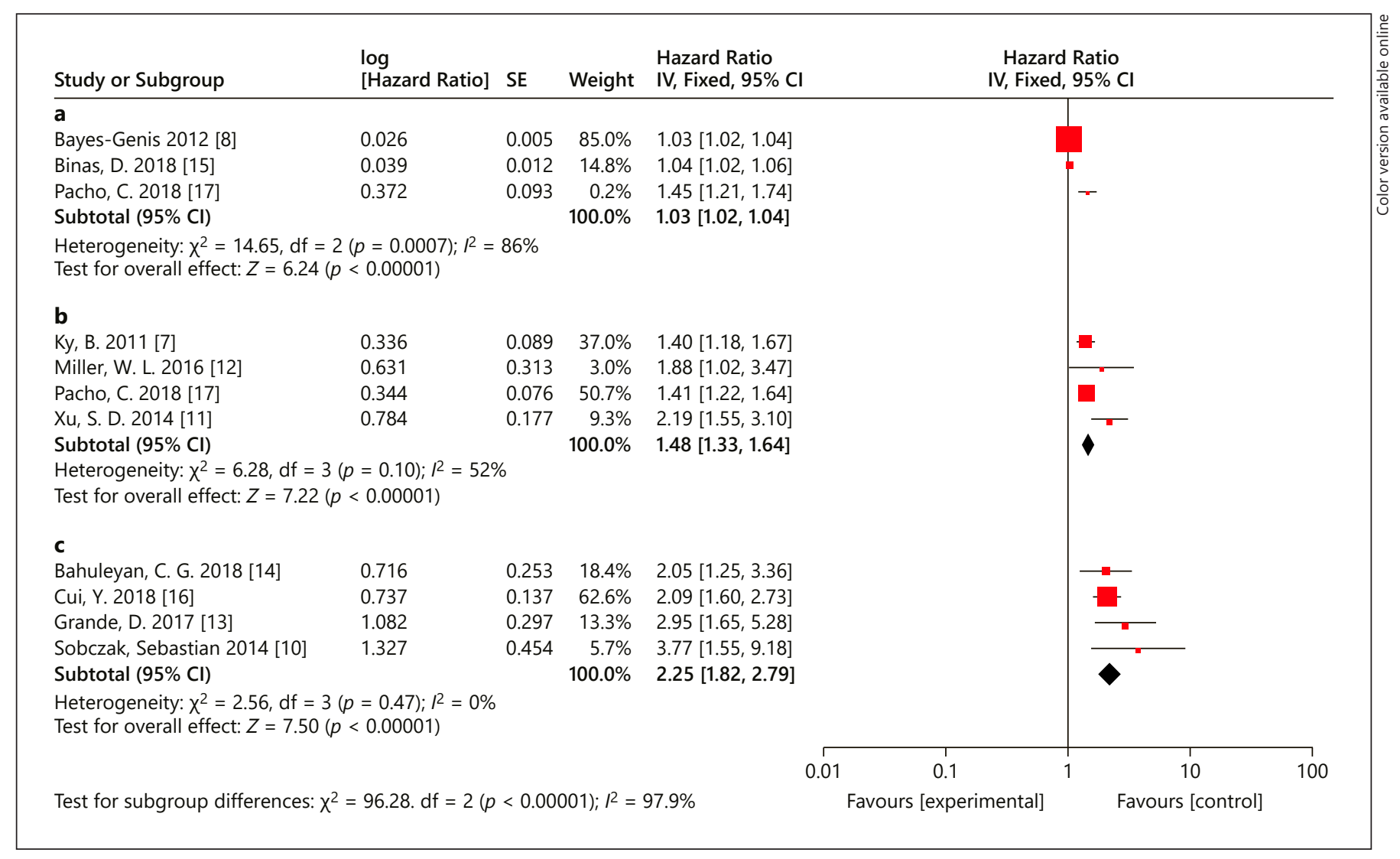

Fig. 2. Forest plots for the meta-analysis of long-term prognostic value of sST2 concentration in CHF patients with sST2 concentration before sensitivity analysis: ACM (a); ACM/HFR (b); CVM/HFH (c). sST2, soluble suppression of tumorigenicity-2; CHF, chronic heart failure; ACM, all-cause mortality; ACM/HFR, all-cause mortality/ heart failure-related readmission; $\mathrm{CVM} / \mathrm{HFH}$, cardiovascular mortality/heart failure-related hospitalization.

tic efficacy of sST2 concentration for CHF. We extracted HRs for $\log (\mathrm{sST} 2)$ if sST2 concentration was present as continuous variables; we extracted HRs which compared patients from the above to below cutoff value if SST2 concentration was present as categorized variables. We used Cochrane's Q test and $I^{2}$ to evaluated the heterogeneity among the studies. If $I^{2}>50 \%$, which means heterogeneity was significant, sensitivity analyses were used to find out the source of heterogeneity, and the randomized-effects model was used to pool effect sizes. If $I^{2}<50 \%$, the fixed-effects model was used to pool effect sizes. The above statistics are completed by Review Manager (Revman version 5.3; Cochrane Collaboration, Oxford, UK).

\section{Results}

\section{Study Inclusion and Characteristics}

According to the above inclusion criteria, 2,144 studies were retrieved, and 943 duplicated studies were excluded. 1,142 unrelated studies were excluded by reading titles and abstracts; forty-eight articles were excluded after reading the full text, and 11 studies met the criteria of inclusion and exclusion (Fig. 1). The location of the study included Asia, Europe, USA, and multiple areas. The year of publication ranged from 2011 to 2018 , and 5,121 patients in total were included. Three studies reported longterm ACM; four studies reported long-term ACM/HFR; four studies reported long-term $\mathrm{CVM} / \mathrm{HFH}$; and 3 studies reported ACM/HFR (Table 1).

\section{Long-Term Prognostic Endpoints of Meta-Analyses}

Three studies reported the relationship between higher sST2 concertation and ACM (over 1 year after hospitalization) (HR: $1.03,95 \%$ CI: 1.02-1.04, $p=0.0007<0.1, I^{2}=$ $86 \%$; Fig. $2 \mathrm{a}$ ), and 4 studies showed higher concentration of sST2 is related to worse ACM/HFR (over 1 year after hospitalization) (HR: 1.48, 95\% CI: $1.33-1.64, p=0.10$, $I^{2}=52 \%$; Fig. 2b). Four studies showed that the baseline sST2 concentration predicts the risk of CVM/HFH (over 


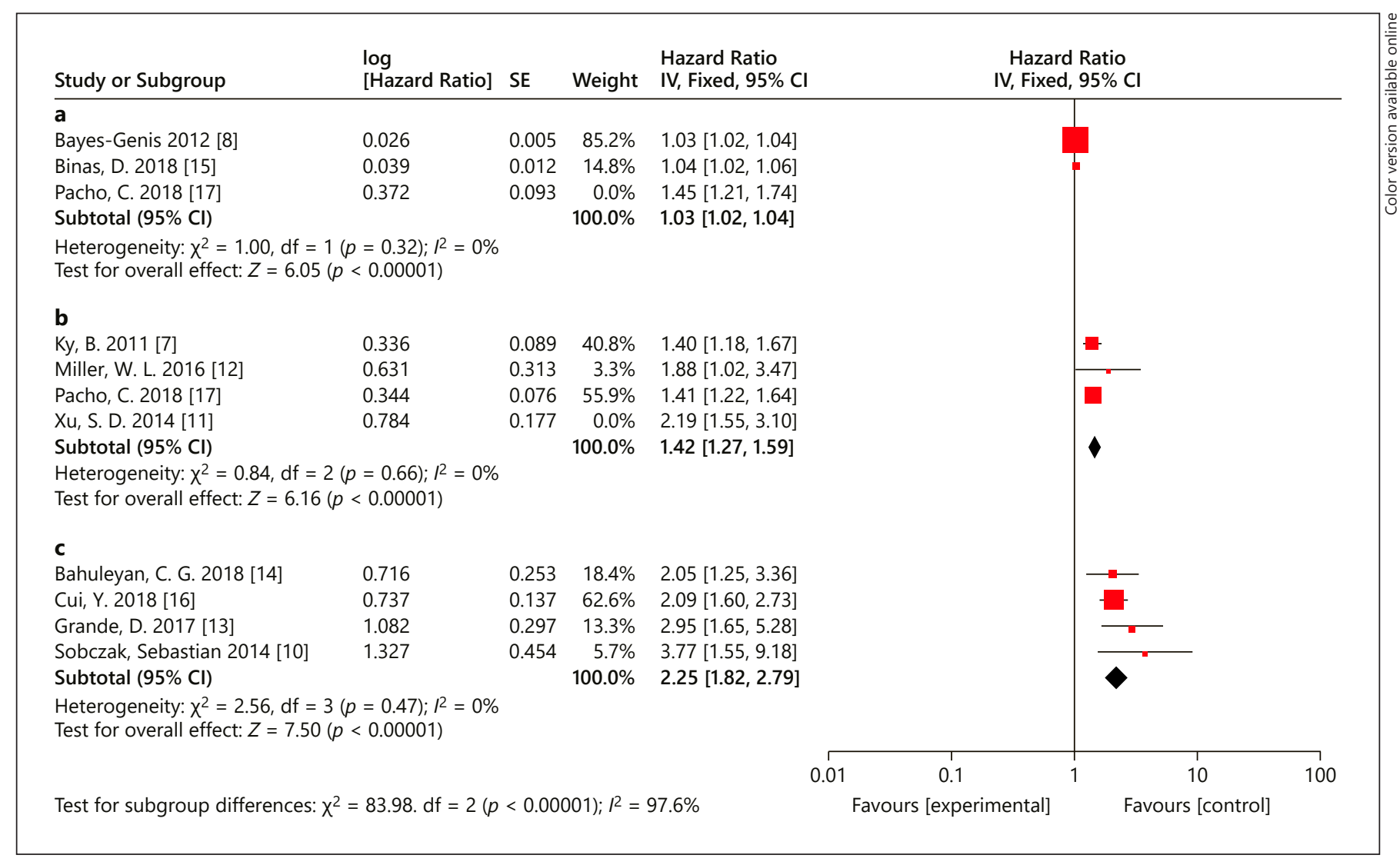

Fig. 3. Forest plots for the meta-analysis of long-term prognostic value of sST2 concentration in CHF patients in the fixed-effect model after sensitivity analysis: ACM (a); ACM/HFR (b); CVM/HFH (c). sST2, soluble suppression of tumorigenicity-2; CHF, chronic heart failure; ACM, all-cause mortality; ACM/HFR, all-cause mortality/ heart failure-related readmission; $\mathrm{CVM} / \mathrm{HFH}$, cardiovascular mortality/heart failure-related hospitalization.

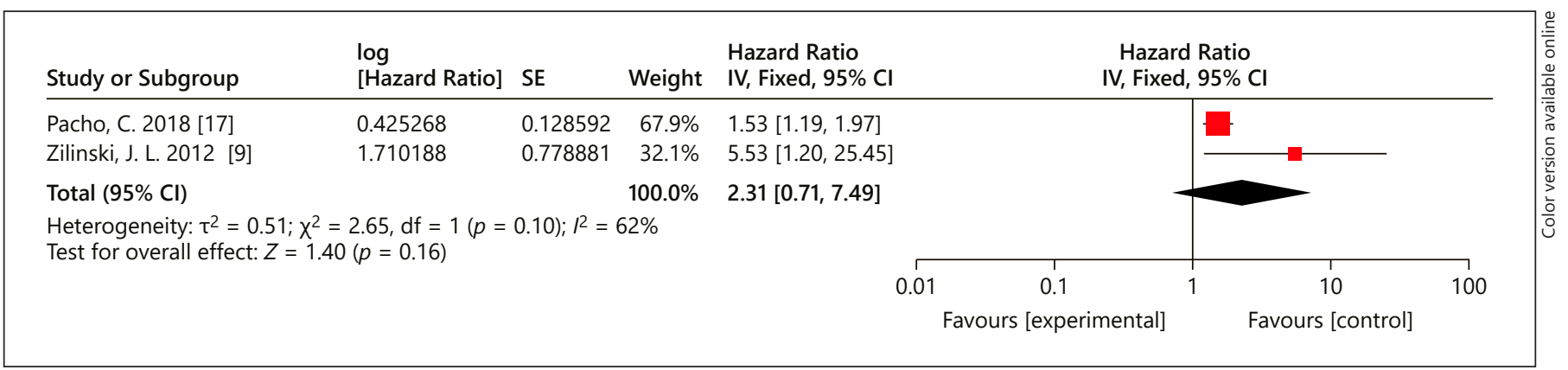

Fig. 4. Forest plots for the meta-analysis of short-term prognostic value of sST2 concentration in CHF patients in the randomized-effect model. sST2, soluble suppression of tumorigenicity-2; CHF, chronic heart failure.

1 year after hospitalization) (HR: 2.25, 95\% CI: 1.82-2.79, $p=0.470, I^{2}=0 \%$; Fig. $2 c$ ) and had no significant heterogeneity. The first two of analyses of endpoints had a significant heterogeneity, and thus sensitivity analyses were needed to find the reason of heterogeneity. The sensitivity analyses showed that after omitting the study by Pacho et al. [17] and Xu et al. [11], the heterogeneity of long-term ACM and long-term ACM/HFR became nonsignificant. Besides, there was no change after omitting any of the studies in the analysis of long-term CVM/HFH. There- 
fore, the fixed-effect model was used. As shown in the fixed-effect model, higher sST2 concertation still predicted worse long-term ACM (HR: 1.03, 95\% CI: 1.02-1.04, $p=0.32, I^{2}=0 \%, Z=6.24$; Fig. 3a), long-term ACM/HFR (HR: 1.42 , 95\% CI: $1.27-1.59, p=0.66, I^{2}=0 \%, Z=7.22$; Fig. 3b), and long-term CVM/HFH (HR: 2.25, 95\% CI: 1.82-2.79, $p=0.47, I^{2}=0 \%, Z=7.50$; Fig. 3c). The publication biases of the meta-analyses because of limited number of included studies could not be performed.

\section{Short-Term Prognostic Endpoints of Meta-Analyses}

Two studies reported the risk of ACM/HFR in short term (1-1.5 months). Because of the significant heterogeneity and limited number of studies, the randomized-effects model was performed. The pooled analysis of these studies showed no significant difference in risk of ACM/ HFR between people with different concentrations of sST2 in baseline risk of ACM/HFR (HR: 2.31, 95\% CI: $0.71-7.49, p=0.10, I^{2}=62 \%, Z=1.46$; Fig. 4 ). The publication biases of the meta-analyses because of limited number of included studies could not be performed.

\section{Discussion/Conclusion}

In this meta-analysis, 11 prospective and post hoc studies from different regions and countries were integrated. The survival data of 5,121 patients were included. The results showed that sST2 is related to long-term ACM, ACM/HFR, and CVM/HFH. The sensitivity analysis and fixed-effect model analysis proved that the results were stable and reliable, which provided evidence from evidence-based medicine for sST2 as a clinical prognosis index.

2016 ESC Guidelines recommended LVEF as indicators to evaluate the severity of ventricular remodeling and cardiac function, but there are still great limitations when it comes to clinical application [21].The guidelines also have mentioned that the natriuretic peptide plays an important role in the diagnosis and management of heart failure. However, 1 study pointed that NT-proBNP does not have a significant relationship with measures of cardiac function and exercise tolerance [22]. Also, BNP and NT-proBNP are cleared by the kidney, and their concentration is correlated similarly to glomerular filtration rate [23]. Besides, it is hard to identify the presence of change in filling pressures according to the change of NPs, and the diagnose of early decompensation should be dependent on the combination of NP concentration, weight gain, and symptoms [24].
sST2 is one of the subtypes of ST2 [25]. Compared to the natriuretic peptide, concentration of sST2 seems to be not affected by age, gender, and kidney function and has been known as the HbAlc of heart failure [26]. In the context of stretch-induced injury to the heart, sST2 prevents signaling through ST2L by the binding of IL-33 to sST2 and thus inhibiting the cardioprotective effect [27, 28]. In this way, it has been proved to be significantly related to the prognosis of cardiovascular disease [29]. Because of the low coefficient of variation in the concentration, sST2 has a great potential in serial measurement of HF [30]. sST2 and NT-proBNP complement each other in the risk stratification of heart failure [31]. This combination of biomarkers will also affect clinical decisionmaking in the future [32].

The strength of our study is using HRs after adjustment for multiple baseline covariates in the analysis of the relationship between the concentration of sST2 and the prognosis of CHF. Two recent meta-analyses [33, 34] did a remarkable job to reveal the prognostic value of sST2 in acute heart failure and CHF independently. What is different from these 2 studies is that our paper reveals the role of sST2 in CHF from 2 aspects: shortterm effect and long-term effect. Furthermore, we have studied more and different clinical endpoints (ACM, $\mathrm{ACM} / \mathrm{HFR}$, and CVM/HFH) to elucidate the important role of sST2 in the prognosis of CHF. This article will promote people's cognition of the prognostic role of sST2 in heart failure. There are some limitations and deficiencies in our study. Firstly, it is difficult to determine the stability of this study through the publication bias due to the small number of studies included, and thus need to be performed, especially, in the aspect of shortterm endpoints. Secondly, the study by Ky et al. [7] has larger sample size than others, and the 2 studies $[9,17]$ in the analysis for short-term ACM/HFR have great difference in sample size and that may lead to bias. Last but not least, our study does not restrict the measurement of sST2. It has been proved that compared with other detection methods, the Pressure ${ }^{\circledR}$ ST2 assay can determine the concentration of sST2 more accurately $[35,36]$. However, different detection methods are included in our study, which will also interfere with the stability and accuracy of this study.

In conclusion, our study showed that higher concentration of sST2 can be used as independent prognostic factors for $\mathrm{CHF}$ and is suitable for long-term endpoints (ACM, ACM/HFR, and CVM/HFH), regardless of shortterm ACM/HFR, and more studies need to be performed in this aspect. This meta-analysis supports the wide ap- 
plication of sST2 in CHF and provides a supplement for the natriuretic peptide and other biomarkers of heart failure risk stratification.

\section{Statement of Ethics}

The authors have no ethical conflicts to disclose.

\section{Conflict of Interest Statement}

The authors have no conflicts of interest to declare.

\section{Funding Sources}

This study was supported by grants from the Priority Academic Program Development of Jiangsu Higher Education Institutions (2019YSHL051).

\section{Author Contributions}

Supervision: Hao Chen and Yihuang Gu. Data curation, investigation, and methodology: Guoqi Dong and Hongru Zhang. Software: Guoqi Dong. Writing - original draft: Guoqi Dong.

\section{References}

1 Chioncel O, Lainscak M, Seferovic PM, Anker SD, Crespo-Leiro MG, Harjola VP, et al. Epidemiology and one-year outcomes in patients with chronic heart failure and preserved, midrange and reduced ejection fraction: an analysis of the ESC Heart Failure Long-Term Registry. Eur J Heart Fail. 2017 Dec;19(12):1574-85.

2 Ziaeian B, Fonarow GC. Epidemiology and aetiology of heart failure. Nat Rev Cardiol. 2016 Jun;13(6):368-78.

3 Kajstura J, Rota M, Cappetta D, Ogórek B, Arranto $\mathrm{C}$, Bai $\mathrm{Y}$, et al. Cardiomyogenesis in the aging and failing human heart. Circulation. 2012 Oct 9;126(15):1869-81.

4 Travers JG, Kamal FA, Robbins J, Yutzey KE, Blaxall BC. Cardiac fibrosis: the fibroblast awakens. Circ Res. 2016 Mar 18;118(6):102140.

5 Kotsiou OS, Gourgoulianis KI, Zarogiannis SG. IL-33/ST2 axis in organ fibrosis. Front Immunol. 2018;9:2432

6 Yancy CW, Jessup M, Bozkurt B, Butler J, Casey DE Jr, Colvin MM, et al. 2017 ACC/ AHA/HFSA focused update of the 2013 ACCF/AHA guideline for the management of heart failure: a report of the American College of Cardiology/American Heart Association task force on clinical practice guidelines and the Heart Failure Society of America. J Am Coll Cardiol. 2017 Aug 8;70(6):776-803.

7 Ky B, French B, McCloskey K, Rame JE, McIntosh E, Shahi P, et al. High-sensitivity ST2 for prediction of adverse outcomes in chronic heart failure. Circ Heart Fail. 2011 Mar;4(2): $180-7$.

8 Bayes-Genis A, de Antonio M, Galán A, Sanz $\mathrm{H}$, Urrutia A, Cabanes R, et al. Combined use of high-sensitivity ST2 and NTproBNP to improve the prediction of death in heart failure. Eur J Heart Fail. 2012 Jan;14(1):32-8.

9 Zilinski JL, Shah RV, Gaggin HK, Gantzer ML, Wang TJ, Januzzi JL. Measurement of multiple biomarkers in advanced stage heart failure patients treated with pulmonary artery catheter guided therapy. Crit Care. 2012 Jul 25;16(4):R135.
10 Sobczak S, Wojtczak-Soska K, Ciurus T, Sakowicz A, Pietrucha T, Lelonek M. Single sST2 protein measurement predicts adverse outcomes at 1-year follow-up in patients with chronic heart failure. Pol Arch Med Wewn. 2014;124(9):452-8.

11 Xu SD, Su GH, Lu YX, Shuai XX, Tao XF, Meng YD, et al. Elevated soluble ST2 and depression increased the risk of all-cause mortality and hospitalization in patients with heart failure. Int Heart J. 2014;55(5):445-50.

12 Miller WL, Saenger AK, Grill DE, Slusser JP, Bayes-Genis A, Jaffe AS. Prognostic value of serial measurements of soluble suppression of tumorigenicity 2 and galectin- 3 in ambulatory patients with chronic heart failure. J Card Fail. 2016 Apr;22(4):249-55.

13 Grande D, Leone M, Rizzo C, Terlizzese P, Parisi G, Gioia MI, et al. A multiparametric approach based on NT-proBNP, ST2, and galectin-3 for stratifying one year prognosis of chronic heart failure outpatients. J Cardiovasc Dev Dis. 2017 Jul 15;4(3):9.

14 Bahuleyan CG, Alummoottil GK, Abdullakutty J, Lordson AJ, Babu S, Krishnakumar VV, et al. Prognostic value of soluble ST2 biomarker in heart failure patients with reduced ejection fraction: a multicenter study. Indian Heart J. 2018 Jul;70(Suppl 1):S79-84.

15 Binas D, Daniel H, Richter A, Ruppert V, Schlüter KD, Schieffer B, et al. The prognostic value of sST2 and galectin- 3 considering different aetiologies in non-ischaemic heart failure. Open Heart. 2018;5(1):e000750.

16 Cui Y, Qi X, Huang A, Li J, Hou W, Liu K. Differential and predictive value of galectin- 3 and soluble suppression of tumorigenicity-2 (sST2) in heart failure with preserved ejection fraction. Med Sci Monit. 2018 Jul 24;24:5139-46.

17 Pacho C, Domingo M, Núñez R, Lupón J, Núñez J, Barallat J, et al. Predictive biomarkers for death and rehospitalization in comorbid frail elderly heart failure patients. BMC Geriatr. 2018 May 9;18(1):109.

18 Stroup DF, Berlin JA, Morton SC, Olkin I, Williamson GD, Rennie D, et al. Meta-anal- ysis of observational studies in epidemiology: a proposal for reporting. Meta-analysis of Observational Studies in Epidemiology (MOOSE) group. JAMA. 2000 Apr 19; 283(15):2008-12.

19 Parmar MK, Torri V, Stewart L. Extracting summary statistics to perform meta-analyses of the published literature for survival endpoints. Stat Med. 1998 Dec 30;17(24):281534.

20 Margulis AV, Pladevall M, Riera-Guardia N, Varas-Lorenzo C, Hazell L, Berkman ND, et al. Quality assessment of observational studies in a drug-safety systematic review, comparison of two tools: the Newcastle-Ottawa Scale and the RTI item bank. Clin Epidemiol. 2014;6:359-68.

21 Ponikowski P, Voors AA, Anker SD, Bueno H, Cleland JG, Coats AJ, et al. 2016 ESC Guidelines for the diagnosis and treatment of acute and chronic heart failure: the task force for the diagnosis and treatment of acute and chronic heart failure of the European Society of Cardiology (ESC). Developed with the special contribution of the Heart Failure Association (HFA) of the ESC. Eur J Heart Failure. 2016 Aug; 18(8):891-975.

22 Parovic M, Okwose NC, Bailey K, Velicki L, Fras Z, Seferovic PM, et al. NT-proBNP is a weak indicator of cardiac function and haemodynamic response to exercise in chronic heart failure. ESC Heart Failure. 2019 Apr; 6(2):449-54.

23 van Kimmenade RR, Januzzi JL Jr, Bakker JA, Houben AJ, Rennenberg R, Kroon AA, et al. Renal clearance of B-type natriuretic peptide and amino terminal pro-B-type natriuretic peptide a mechanistic study in hypertensive subjects. J Am Coll Cardiol. 2009 Mar 10; 53(10):884-90.

24 Mueller C, McDonald K, de Boer RA, Maisel A, Cleland JGF, Kozhuharov N, et al. Heart Failure Association of the European Society of Cardiology practical guidance on the use of natriuretic peptide concentrations. Eur J Heart Failure. 2019 Jun;21(6):715-31. 
25 De la Fuente M, MacDonald TT, Hermoso MA. The IL-33/ST2 axis: role in health and disease. Cytokine Growth Factor Rev. 2015 Dec;26(6):615-23.

26 Maisel AS, Di Somma S. Do we need another heart failure biomarker: focus on soluble suppression of tumorigenicity 2 (sST2). Eur Heart J. 2017 Aug 7;38(30):2325-33.

27 Sanada S, Hakuno D, Higgins LJ, Schreiter ER, McKenzie AN, Lee RT. IL-33 and ST2 comprise a critical biomechanically induced and cardioprotective signaling system. J Clin Invest. 2007 Jun;117(6):1538-49.

28 Moore SA, Januzzi JL Jr. Found in translation soluble ST2 and heart disease. J Am Coll Cardiol. 2010 Jan 19;55(3):251-3.

29 Altara R, Ghali R, Mallat Z, Cataliotti A, Booz GW, Zouein FA. Conflicting vascular and metabolic impact of the IL-33/sST2 axis. Cardiovascular Res. 2018 Oct 1;114(12):1578-94.
30 Bayes-Genis A, Nunez J, Lupon J. Soluble ST2 for prognosis and monitoring in heart failure: the new gold standard? J Am Coll Cardiol. 2017 Nov 7;70(19):2389-92.

31 Pascual-Figal DA, Manzano-Fernández S, Boronat M, Casas T, Garrido IP, Bonaque JC, et al. Soluble ST2, high-sensitivity troponin $\mathrm{T}$ - and N-terminal pro-B-type natriuretic peptide: complementary role for risk stratification in acutely decompensated heart failure. Eur J Heart Fail. 2011 Jul;13(7):718-25.

32 Pascual-Figal DA, Ordoñez-Llanos J, Tornel PL, Vázquez R, Puig T, Valdés M, et al. Soluble ST2 for predicting sudden cardiac death in patients with chronic heart failure and left ventricular systolic dysfunction. J Am Coll Cardiol. 2009 Dec 1;23):2174-9.

33 Aimo A, Vergaro G, Passino C, Ripoli A, Ky B, Miller WL, et al. Prognostic value of soluble suppression of tumorigenicity-2 in chronic art failure: a meta-analysis. JACC Heart Fail. 2017 Apr 1;5(4):280-6.
34 Aimo A, Vergaro G, Ripoli A, Bayes-Genis A, Pascual Figal DA, de Boer RA, et al. Metaanalysis of soluble suppression of tumorigenicity- 2 and prognosis in acute heart failure. JACC Heart Fail. 2017 Apr 1;5(4):287-96.

35 Mueller T, Zimmermann M, Dieplinger B, Ankersmit HJ, Haltmayer M. Comparison of plasma concentrations of soluble ST2 measured by three different commercially available assays: the MBL ST2 assay, the Presage ST2 assay, and the R\&D ST2 assay. Clin Chim Acta. 2012 Oct 9;413(19-20):1493-4.

36 Mueller T, Dieplinger B. The Presage ${ }^{\circ}$ ST2 assay: analytical considerations and clinical applications for a high-sensitivity assay for measurement of soluble ST2. Expert Rev Mol Diagn. 2013 Jan;13(1):13-30. 